\title{
Adaptive Optimization Framework for Control of Multi-Agent Systems
}

\author{
Anna Lukina \\ Technische Universitát Wien \\ Treitlstrasse 3, 1040, Vienna, Austria \\ +43(677)62044339, anna.lukina@tuwien.ac.at \\ http://logic-cs.at/phd/students/anna-lukina/
}

\begin{abstract}
The main focus of this work is an optimization-based framework for control of multi-agent systems that synthesizes actions steering a given system towards a specified state. The primary motivation for the research presented is a fascination with birds, which save energy on long-distance flights via forming a $\mathrm{V}$-shape. We ask the following question: Are V-formations a result of solving an optimization problem and can this concept be utilized in multi-agent systems, particularly in drones swarms, to increase their safety and resilience? We demonstrate that our framework can be applied to any system modeled as a controllable Markov decision process with a cost (reward) function. A key feature of the procedure we propose is its automatic adaptation to the performance of optimization towards a given global objective. Combining model-predictive control and ideas from sequential MonteCarlo methods, we introduce a performance-based adaptive horizon and implicitly build a Lyapunov function that guarantees convergence. We use statistical model-checking to verify the algorithm and assess its reliability.
\end{abstract}

\section{Taking Off}

Airplane industry is making every effort to lower their fuel costs by as little as one percent. In August 2017, Boeing Co. and NASA announced a collaboration on reducing fuel consumption of commercial flights by copying the ways in which migratory birds successfully perform long-distance flights (Bloomberg 2017). They do so by taking advantage of the upward air boost generated under the flapping wings of their flock mates while the leader does most of the work (Weimerskirch et al. 2001).

This line of research is ongoing and Boeing is actively striving to collect evidence showing an expected ratio of saved fuel for commercial aircraft. NASA, in turn, has been long studying efficient nature-inspired approaches to flights in the Earth's atmosphere and outer space (NASA 2003). Their experiments with Boeing C-17 military transport planes (Forse 2013) and F-18 fighter jets (NASA 2001) flying in formation showed an energy saving of at least 10 and 15 percent, respectively. The core of this work was inspired by these discoveries and designed to improve state-ofthe-art techniques for control of stochastic multi-agent systems (MAS) in general.

Copyright (C) 2019, Association for the Advancement of Artificial Intelligence (www.aaai.org). All rights reserved.
In this work, we aim to design a real-time adaptive optimization framework for the resilient formation control of unmanned aerial vehicles in adversary environmental conditions. We envision that a bird-inspired optimization technique can help a collection of autonomous agents fly safely and efficiently while maintaining formation.

The physical environment renders the problem of MAS control extremely cumbersome. Due to a wealth of uncertainties introduced by physical processes, the system is best described by stochastic models. Approximate prediction techniques, such as statistical model checking (SMC), have therefore recently become increasingly popular (Grosu and Smolka 2005; Clarke and Zuliani 2011). Controlling the systems, that is, computing appropriate response actions depending on the environment, involves probabilistic state estimation, as well as optimal action prediction, i.e., choosing the best next step by simulating the future. In this work, we develop a general framework addressing questions of control of multi-agent systems under uncertainty via optimization.

\section{Ground Control}

The main contribution of this work is a general adaptive optimization framework for control of MAS. Every bird-like agent in our model moves in 2-dimensional space locally governed by the same control law (Yang et al. 2016). Any agent in the flock can detect the positions and velocities of all other agents through sensors. Given this information, the agent's controller calculates an optimal acceleration based on the three metrics we define: clear view, velocity matching, and upwash benefit. Formulated this way the optimization problem we solve leads to a V-formation as an optimal state for the flock.

Ultimately, we synthesize an algorithm providing analytical guarantees of birds getting into a V-formation starting from a random configuration using a flying drones simulation model and statistical model checking (Lukina et al. 2017; Tiwari et al. 2017). Particle swarm optimization (Kennedy and Eberhart 1995) uniformly distributes the particles in space and adjusts their velocities to lead the swarm to satisfying a given property while using a random factor in the adjustment rule in order to explore the space.

The resulting V-formation provides the birds with a clear view of the front field and visibility of their lateral neighbors. Moreover, the formation is of great importance to 


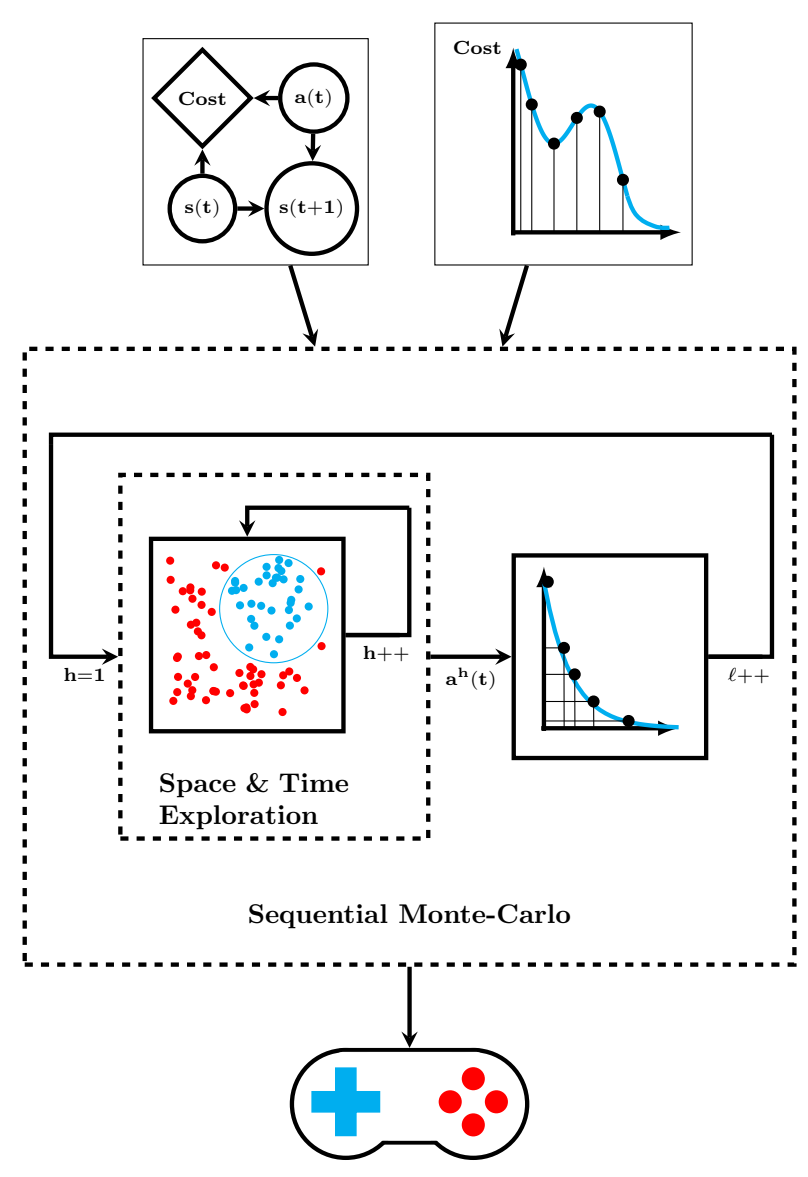

Figure 1: Main components of the adaptive optimization framework. Arrows direct the process flow of the algorithm.

flocking birds for saving energy from the free lift as a beneficial effect of the upwash region generated off the trailing edge of wings of the birds in front of them (Weimerskirch et al. 2001). We believe this approach can lead to a breakthrough discovery in developing energy-efficient and reliable autonomous technologies.

Due to stochastic nature and large scale of the systems we are interested in, the most promising sources of answers to the questions we pose are approximate algorithms, metaheuristic techniques, and optimization-based approaches to control. Experimenting with existing algorithms in application to our model inspired us to develop a general optimization framework, self-adaptive and flexible for the user to specify a problem setting and properties of their interest.

The block-diagram in Figure 1 comprises main components of the framework and its process flow. In brief, the core procedure is enclosed in the outer dashed block, which performs level-by-level adaptive model-predictive control inspired by sequential Monte-Carlo methods. It receives a controllable stochastic multi-agent system on the top left and a cost function on the top right as inputs. Resulting from the space-time exploration using particle swarm optimization, if the minimal cost can be reached, the framework outputs a controller driving the system towards optimal state. Otherwise, it provides statistical guarantees that no path exists.

\section{Landing}

Regarding deployment, we see the following approach. The framework can be implemented as a local controller on each drone and communication will require broadcasting positions and output of the algorithm to other drones in the neighborhood through a shared memory. In this case, a central agent will be needed to periodically compute the global cost. We plan to use OpenUAV (Schmittle et al. 2018) to test the framework on drone formation control scenarios.

Acknowledgments. The author would like to thank Emir Demirović for very valuable feedback, and her supervisor Radu Grosu. This work was partially supported by the Doctoral Program Logical Methods in Computer Science and RiSE/SHiNE (S11405-N23 and S11412-N23) project funded by the Austrian Science Fund (FWF) project W1255N23, and National Science Foundation grant CCF 1423296.

\section{References}

Bloomberg. 2017. Boeing Copies Flying Geese to Save Fuel.

Clarke, E., and Zuliani, P. 2011. Statistical model checking for cyber-physical systems. In ATVA 2011, volume 6996 of LNCS, 1-12. Springer.

Forse, U. A. 2013. Inspired by nature: Innovative C-17 flight tests to save AF millions.

Grosu, R., and Smolka, S. 2005. Monte Carlo model checking. In TACAS 2005, volume 3440 of LNCS, 271-286. Springer Verlag.

Kennedy, J., and Eberhart, R. 1995. Particle swarm optimization.

Lukina, A.; Esterle, L.; Hirsch, C.; Bartocci, E.; Yang, J.; Tiwari, A.; Smolka, S. A.; and Grosu, R. 2017. ARES: Adaptive Receding-Horizon Synthesis of Optimal Plans. In TACAS 2017, volume 10206 of LNCS, 286-302.

NASA. 2001. F/A-18 Autonomous Formation Flight (AFF). NASA. 2003. Fly Like a Bird.

Schmittle, M.; Lukina, A.; Vacek, L.; Das, J.; van Buskirk, C. P.; Rees, S.; Sztipanovits, J.; Grosu, R.; and Kumar, V. 2018. OpenUAV: a UAV testbed for the CPS and robotics community. In ICCPS 2018, 130-139. IEEE / ACM.

Tiwari, A.; Smolka, S. A.; Esterle, L.; Lukina, A.; Yang, J.; and Grosu, R. 2017. Attacking the V: On the Resiliency of Adaptive-Horizon MPC. In ATVA 2017, volume 10482 of LNCS, 446-462. Springer.

Weimerskirch, H.; Martin, J.; Clerquin, Y.; Alexandre, P.; and Jiraskova, S. 2001. Energy saving in flight formation. Nature 413(6857):697-698.

Yang, J.; Grosu, R.; Smolka, S. A.; and Tiwari, A. 2016. Love Thy Neighbor: V-Formation as a Problem of Model Predictive Control. In CONCUR 2016, volume 59 of LIPICs, 4:1-4:5. 